\title{
Melatonin and sleep responses to normobaric hypoxia and aerobic physical exercise: A randomized controlled trial
}

\author{
Valdir de Aquino Lemos ${ }^{\mathrm{a}, \mathrm{b}}$, Ronaldo Vagner Thomatieli dos Santos ${ }^{\mathrm{e}}$, \\ Hanna Karen Moreira Antunes ${ }^{\mathrm{e}}$, Claus Behn ${ }^{\mathrm{f}, \mathrm{g}}$, Gines Viscor ${ }^{\mathrm{h}}$, Fabio Santos Lira ${ }^{\mathrm{d}}$, \\ Irene Guarido Luz Bittar ${ }^{\mathrm{a}}$, Aline Venticinque Caris ${ }^{\mathrm{a}}$, Sergio Tufik ${ }^{\mathrm{a}}$, Marco Tulio De Mello ${ }^{\mathrm{a}, \mathrm{c}, *}$ \\ ${ }^{a}$ Department of Psychobiology, Universidade Federal de São Paulo (UNIFESP), São Paulo, Brazil \\ ${ }^{\mathrm{b}}$ Department of Psychology, Universidade Braz Cubas (UBC), Mogi das Cruzes, Brazil \\ ${ }^{\mathrm{c}}$ Sports Department, Universidade Federal de Minas Gerais (UFMG), Belo Horizonte, Brazil \\ ${ }^{\mathrm{d}}$ Exercise and Immunometabolism Research Group, Department of Physiology and Education, Universidade do Estado de São Paulo (UNESP), Presidente Prudente, Brazil \\ e Department of Biosciences, Universidade Federal de São Paulo (UNIFESP), São Paulo, Brazil \\ ${ }^{\mathrm{f}}$ Program of Physiology and Biophysics, ICBM, Faculty of Medicine, University of Chile, Santiago, Chile \\ ${ }^{\mathrm{g}}$ Gerencia Gestión del Conocimiento, Mutual de Seguridad, CChC, Santiago, Chile \\ ${ }^{\mathbf{h}}$ Department of Physiology and Immunology, University of Barcelona, Barcelona, Spain
}

\section{A R T I C L E I N F O}

\section{Keywords:}

Hypoxia

Exercise

Melatonin

\begin{abstract}
A B S T R A C T
This work evaluated the effects of moderate physical exercise performed under hypoxic conditions on melatonin and sleep. Forty healthy men were randomized into four groups: Normoxia $(\mathrm{N})(n=10)$; Hypoxia $(\mathrm{H})(\mathrm{n}=10)$; Exercise under Normoxia (EN) $(n=10)$; and Exercise under Hypoxia $(E H)(n=10)$. The observation period for all groups was approximately $36 \mathrm{~h}$, beginning with a first night devoid of any intervention. Aerobic exercise was performed by the EN and $\mathrm{EH}$ groups on a treadmill at $50 \%$ of the ventilatory threshold intensity for $60 \mathrm{~min}$. Sleep evaluation was performed on the 1st and 2nd nights. Venous blood samples for the melatonin measurement were obtained on the 1st and 2nd days at 7:30 AM as well as on the 1st and 2nd nights at 10:30 PM. On the 2nd night, melatonin was higher in the $\mathrm{H}$ group than in the $\mathrm{N}$ group, but both were lower than values of the EH group. The nocturnal increase in melatonin was inversely correlated with the oxygen saturation of hemoglobin $\left(\mathrm{SaO}_{2} \%\right)$ on the 2nd night in the $\mathrm{H}$ group and on the 2nd day in the $\mathrm{EH}$ group. Diurnal remission of nocturnal melatonin appeared to be postponed in the $\mathrm{H}$ group and even more so in the $\mathrm{EH}$ group. Thus, normobaric hypoxia, which is equivalent to oxygen availability at an altitude of $4500 \mathrm{~m}$, acutely increases melatonin. Moreover, diurnal remission of the nocturnal increase in melatonin seems to be delayed by hypoxia alone but even more so when acting together with exercise.
\end{abstract}

\section{Introduction}

Melatonin orchestrates the interaction between the central biological clock located in the suprachiasmatic nucleus of the anterior hypothalamus and the gene-anchored peripheral clocks; it may induce sleep in part by promoting adenosine signaling, thus linking circadian and homeostatic control of sleep [1]. As a chemical mediator of darkness and essentially representing an anabolic factor [2], melatonin ( $N$ acetyl-5 methoxytryptamine) is rhythmically secreted in mammals, mainly by the epiphysis [3].

Beyond synchronizing biological clocks [4,5], melatonin also seems to protect against various challenging conditions and functions as a potentially useful therapeutic agent for improving sleep [6], inflammation $[7,8]$, oxidative stress $[9,10,11,12]$, neurohumoral stress [13], and even protection against cancer [14-18].

Hypoxia alters circadian rhythms in rodents $[19,20]$ and in humans [21,22], particularly rhythms inherent to metabolic activity [23]. These changes may have an influence on sleep [24]. The influence of hypoxia on melatonin remains controversial. A study performed with rats exposed to hypobaric hypoxia showed an increase in plasma melatonin [25], whereas plasma melatonin concentrations were decreased in humans following simulated high-altitude exposure in a hypobaric chamber [26]. This hormone acts as an internal indicator of the length of the night, contributes to good 24-h synchronization according to cyclical photoperiod variations, and plays a role in human sleep with respect to core body temperature, particularly during the sleep inducing

\footnotetext{
* Corresponding author at: UFMG, AV. Presidente Carlos Luz, 4664, Pampulha, Belo Horizonte, MG 31.310.250, Brazil.

E-mail address: tmello@demello.net.br (M.T. De Mello).
} 
phase, for example in athletes [27].

Acute physical exercise can influence melatonin serum in normoxia [28]. However, there is some controversy about the effects of physical exercise on the endogenous profile of melatonin secretion [29]. Melatonin levels can increase [30,31], decrease [32,33], or remain unaffected by exercise $[34,35]$. Thus, the present study, which to the best of our knowledge is the first to assess the interaction between exercise, serum melatonin, and sleep under hypoxia, aims to clarify some of these interactions, as the effects of exercise in the hypoxia condition on melatonin concentration is still unknown [36]. Our hypothesis was that exercise may mitigate the effects of hypoxia on sleep which could be useful for athletes, climbers, tourists, workers, and residents in high altitude environments exposed to hypoxia. Thus, the objective of the study was to evaluate the effects of exercise performed in hypoxia at a simulated altitude of $4500 \mathrm{~m}$ on melatonin concentration and sleep.

\section{Materials and methods}

\subsection{Participants}

Forty healthy male volunteers were originally recruited for this study; their characterization data (mean \pm SD) are shown in Table 1 . The participants were randomized into 4 groups: Normoxia $(\mathrm{N})$ $(n=10)$; Hypoxia $(\mathrm{H})(\mathrm{n}=10)$; Exercise under Normoxia (EN) $(n=10)$; and Exercise under Hypoxia $(E H)(n=10)$. Two of the original participants in the $\mathrm{EH}$ group left the study because of severe headaches and vomiting. All volunteers had previously performed activities that included strength training, cycling, and running two or three times a week for at least 6 months; their usual bedtime was approximately $10 \mathrm{p} . \mathrm{m}$. and usual waking time $7 \mathrm{a}$ a.m. All participants were males aged between 20 and 30 years old. The exclusion criteria were: use of tobacco, alcohol or illicit drugs, or medications; exposure to hypoxic conditions in the previous 12 months; and heart problems or sleep disorders, including obstructive sleep apnea, periodic limb movement disorder (PLMD), or any other alterations that could increase sleep fragmentation.

\subsection{Experimental design}

This study was designed in agreement with the guidelines described in the International Declaration of Helsinki 1964 and was approved by the Committee of Ethics in Research at the Federal University of São Paulo - Hospital São Paulo (\# 1110/08). This work was registered in ClinicalTrials.gov; number: (NCT01386814). Fig. 1 summarizes the experimental design. The participants attended the laboratory twice before the main part of the study. On the first visit to the laboratory, the volunteers performed a resting electrocardiogram evaluation as well as an electrocardiogram during the physical effort. On the second visit, an ergospirometric test was performed to determine the $\mathrm{VO}_{\text {2peak }}$ and individual speeds of $50 \%$ of $\mathrm{VO}_{2 \text { peak }}$ for the protocol to be applied in the treadmill exercise. On the third visit, the volunteers were submitted to the experimental procedure according to randomization into four groups.

The volunteers were blinded to the protocol. The $\mathrm{H}$ and $\mathrm{EH}$ groups were exposed for $24 \mathrm{~h}$, from 8:00 AM on the 1st day to 8:00 AM on the 2nd day, to a normobaric decrease in oxygen supply $\left(\mathrm{FiO}_{2}\right.$ of $13.5 \%$ $\mathrm{O}_{2}$ ), equivalent to oxygen availability at an altitude of $4500 \mathrm{~m}$. It was presumed the participants in the $\mathrm{N}$ and EN groups remained at $\mathrm{FiO} 2$ of $20.9 \%$ throughout the study.

On the first night, the volunteers attended the laboratory to undergo the first polysomnography. On the first day, the volunteers in the EN and EH groups participated in a physical exercise session. During the second night, $10 \mathrm{~h}$ after the first physical exercise session and $14 \mathrm{~h}$ after starting the hypoxic condition, the volunteers underwent the second polysomnography. On the second day, at 7:00 a.m. the volunteers awoke after $23 \mathrm{~h}$ in hypoxic conditions. The experiment ended when the volunteers' measurements returned to baseline levels.

Throughout the experiment, each volunteer remained alone in the same room equipped for altitude simulation for $36 \mathrm{~h}$ beginning on the 1 st night and ending on the morning of the 2 nd day. The volunteers had unrestricted access to TV, internet, books, magazines, and a cell phone. Closed-circuit television allowed two-way communication at all times between the participants and staff members. Dinner was served at 7:20 PM and breakfast at 7:40 AM. Lunch lasted from 12:20 to 1:20 PM, followed by a snack at 4:00 PM. The caloric content of the food served was based on the daily energy expenditure of each individual volunteer (mean $1.784 .8 \pm 227.0 \mathrm{kcal} /$ day). Daily energy expenditure was calculated according to [37].

\subsection{Physiological evaluations}

\subsubsection{Normobaric chamber}

The study was conducted in a room equipped for altitude simulations that can reach up to $4500 \mathrm{~m}$, which is equivalent to a barometric pressure of $433 \mathrm{mmHg}$ (normobaric chamber, CAT - Colorado Altitude Training ${ }^{\mathrm{TM}} / 12$ CAT-Air Unit, USA). The normobaric chamber began in a normoxic condition at a $\mathrm{FiO}_{2}$ of $20.9 \% \mathrm{O}_{2}$ and gradually reached a $\mathrm{FiO}_{2}$ of $13.5 \%$, equivalent to $4500 \mathrm{~m}$.

\subsubsection{Oxygen saturation}

Hemoglobin oxygen saturation $\left(\mathrm{SaO}_{2} \%\right)$ was assessed with a finger oximeter (FingerPulse ${ }^{\circledast}$ model MD300C202) during the 1st and 2nd nights at 10:30 PM and during the 1 st and 2nd days at 7:30 AM.

\subsubsection{Physical exercise protocol}

The ergospirometry was performed using the Quark PFT Pulmonary Function Testing - FRC \& DLCO - 4Ergo, Cosmed, Italy. A silicone face mask, Hans Rudolph flow-by face mask (Kansas City, MO, USA), was used for the comfort of the volunteers. This evaluation was performed in normoxic conditions on a treadmill (Life Fitness $9700 \mathrm{HR}^{\circledast}$ - IL, USA) with the speed increased by $1 \mathrm{~km} / \mathrm{h}$ every minute and with an initial load of $3 \mathrm{~min}$ at $6 \mathrm{~km} / \mathrm{h}$. The test ended when the volunteer reached maximum voluntary exhaustion. Throughout the test, a fixed gradient of $1 \%$ was used. The variables evaluated in this study were $\mathrm{VO}_{\text {2peak }}$ and maximum heart rate.

On the days of the experiment, there were two exercise sessions with an intensity of $50 \%$ of $\mathrm{VO}_{2 \text { peak }}$ for $60 \mathrm{~min}$ on a treadmill (Trackmaster ${ }^{\circledR}$ - Maryland, USA) with a slope of $1 \%$. The exercise

Table 1

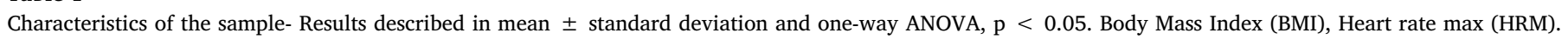

\begin{tabular}{|c|c|c|c|c|c|}
\hline Measures & $\mathrm{N}$ & $\mathrm{H}$ & EN & $\mathrm{EH}$ & $P$ \\
\hline Age (years) & $22 \pm 3$ & $23 \pm 2$ & $26 \pm 3$ & $24 \pm 2$ & 0.06 \\
\hline Body mass $(\mathrm{kg})$ & $69 \pm 1$ & $67 \pm 8$ & $71 \pm 10$ & $72 \pm 11$ & 0.83 \\
\hline Height (m) & $1.78 \pm 0.05$ & $1.75 \pm 0.08$ & $1.75 \pm 0.07$ & $1.78 \pm 0.07$ & 0.83 \\
\hline Energy expenditure (Kcal) & $1757 \pm 141$ & $1718 \pm 158$ & $1751 \pm 160$ & $1858 \pm 140$ & 0,30 \\
\hline BMI $(\mathrm{kg} / \mathrm{m} 2)$ & $21 \pm 7$ & $22 \pm 2$ & $23 \pm 1$ & $22 \pm 1$ & 0.56 \\
\hline HRM (bpm) & $191 \pm 3$ & $194 \pm 8$ & $186 \pm 11$ & $197 \pm 6$ & 0.30 \\
\hline $\mathrm{VO}_{2 \text { peak }}(\mathrm{ml} / \mathrm{kg} / \mathrm{min})$ & $47 \pm 4$ & $47 \pm 6$ & $44 \pm 5$ & $49 \pm 3$ & 0.39 \\
\hline
\end{tabular}



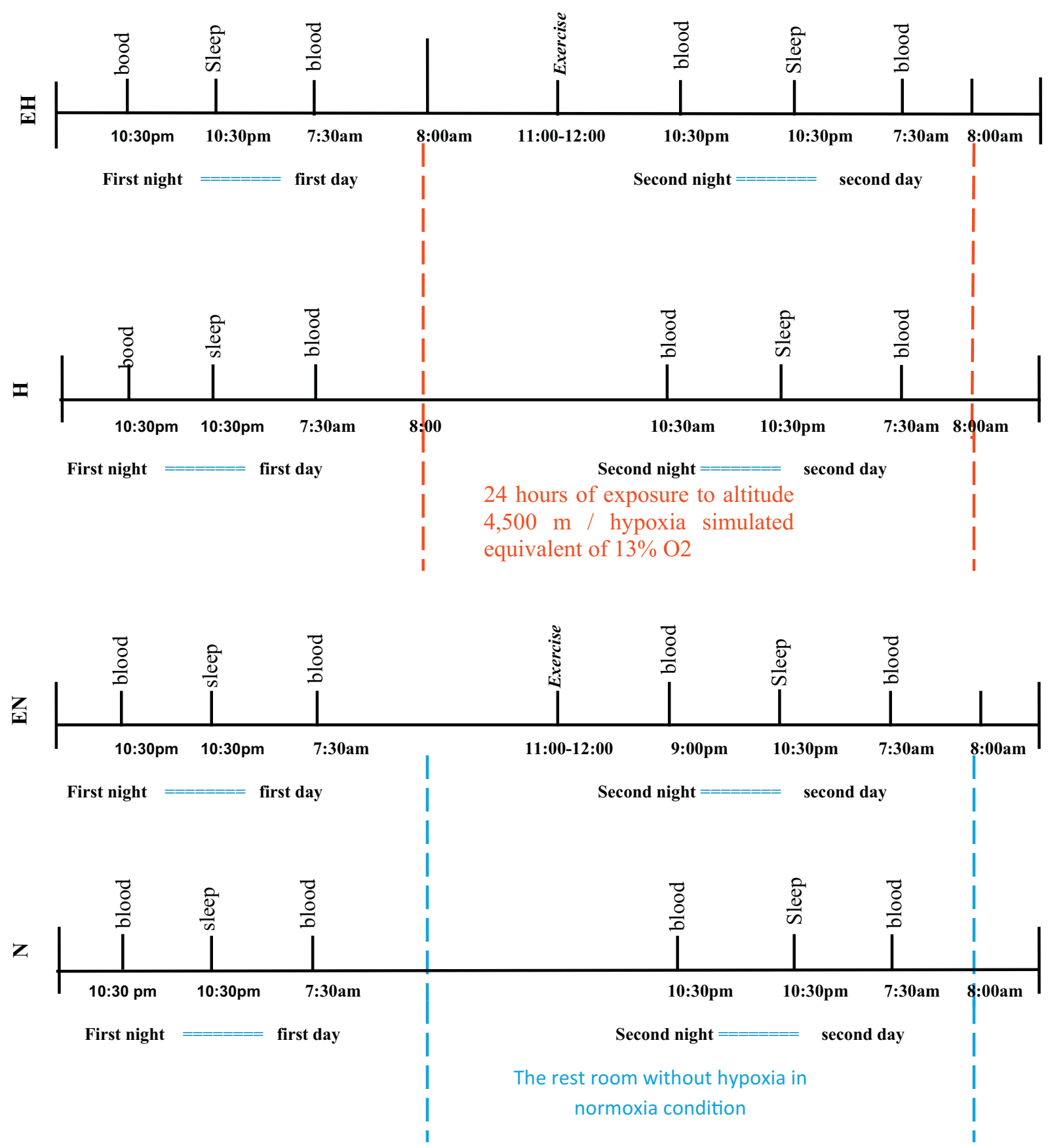

Fig. 1. Summary of the experimental design of all the groups, including (N, H, EN and EH).

began at 11.00 am and finished at 12.00 noon.

\subsubsection{Polysomnography}

All polysomnographies were performed between 10:00 p.m. and 7 a.m. An Embla $N 7000$ polysomnography amplifier with Somnologica Studio version 4 software (Flagahf, Reykjavik, Iceland) was used to assess sleep patterns. Sleep parameters were evaluated according to the criteria described by Iberetal. [38]. The following parameters were assessed: total sleep time (TST) (min), sleep onset latency (min), and sleep efficiency (\%).

\subsection{Laboratory evaluation}

\subsubsection{Plasma melatonin concentration}

Blood samples $(5 \mathrm{~mL})$ were collected from the antecubital vein at four time points: 10:30 p.m. and 7:30 a.m. on the 1st and 2nd nights and the 1st and 2nd days. Remaining supernatant plasma was collected in tubes with $125 \mathrm{IU}$ heparin sodium and centrifuged at $690 \mathrm{x} \mathrm{g}$ for $15 \mathrm{~min}$ at $4^{\circ} \mathrm{C}$, after which the plasma was separated and stored at $-80^{\circ} \mathrm{C}$. The plasma samples were analyzed in duplicate for melatonin concentration by the ELISA method using commercial kits (Wuhan Eiaab Science Co., Ltd.). All measurements followed the manufacturer's specifications and recommendations.

\subsection{Data analysis}

\subsubsection{Statistics}

Data are reported as mean \pm standard deviation and delta $\Delta$. Normality was verified by the Kolmogorov-Smirnov test. A repeated measures and one-way analysis of variance (ANOVA) followed by 
A

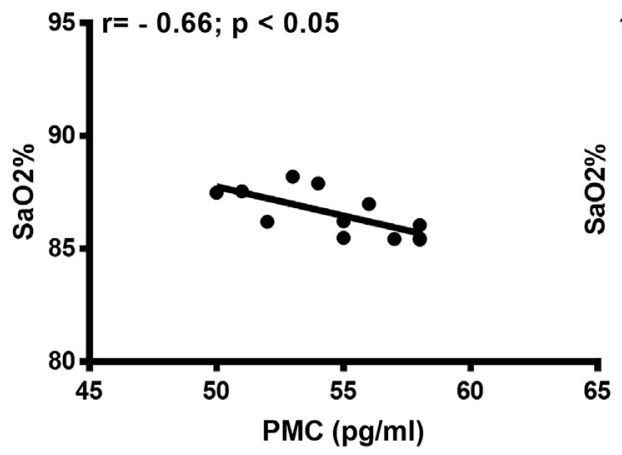

C

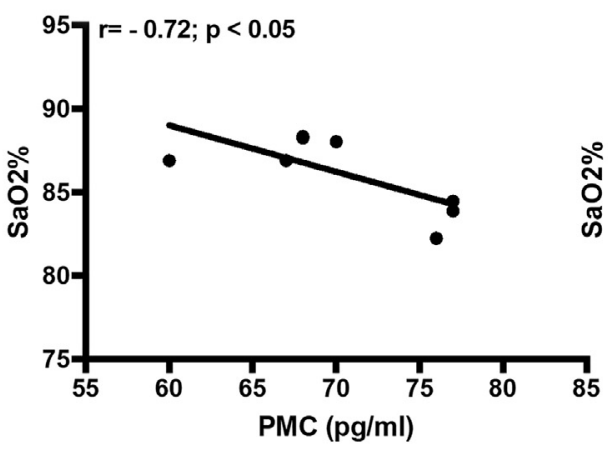

B

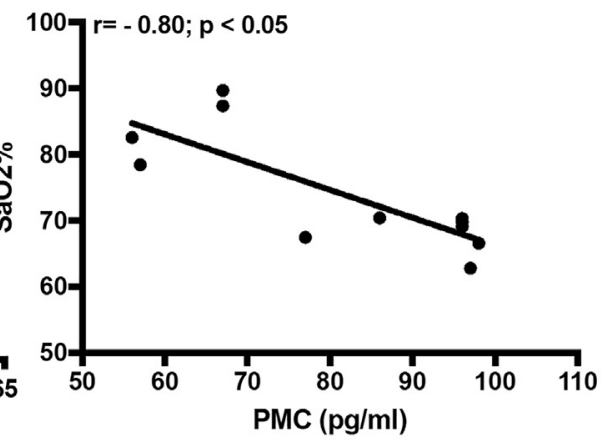

D

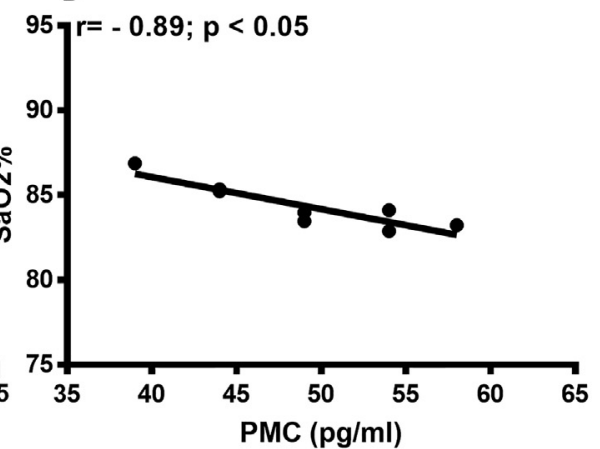

E

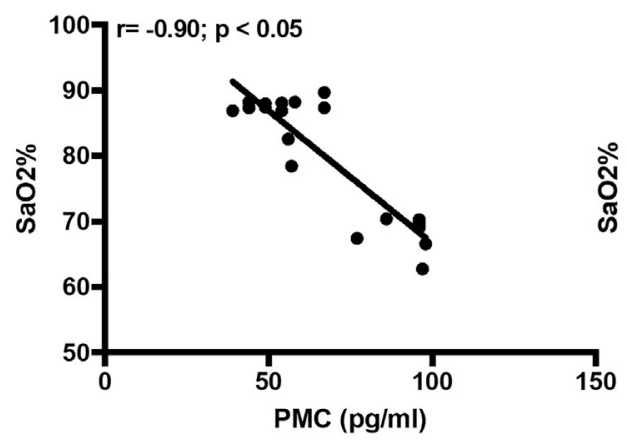

$F$

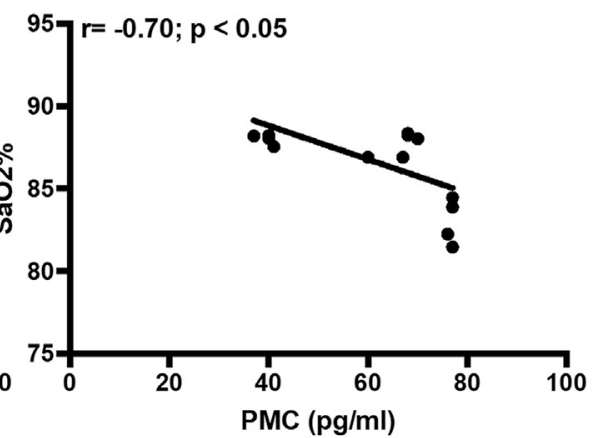

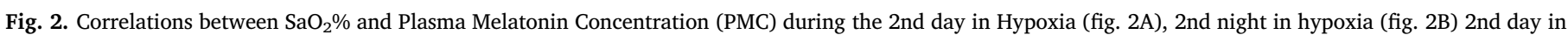

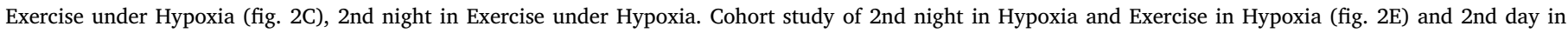
Hypoxia and Exercise in Hypoxia (fig. 2F). Pearson's correlation coefficient ( $r$ ) and $p<0.05$.

Tukey's post hoc test were used to detect significant differences between groups. The correlations of $\mathrm{SaO}_{2}$ with PMC (pg/ml); and PMC $(\mathrm{pg} / \mathrm{ml})$ with SL (min) and SE (\%) were performed using Pearson's correlation coefficient (r). The accepted significance level was $p \leq .05$. Analyses were performed using Statistica ${ }^{\circledR}$ version 6.

\section{Results}

On the 2nd night ( $p<.001$ ) and the 2 nd day, the $\mathrm{SaO}_{2} \%$ was lower in the $\mathrm{H}$ and $\mathrm{EH}$ groups than in the $\mathrm{N}$ and EN groups.

Fig. 2 shows that $\mathrm{SaO}_{2} \%$ and PMC were negatively correlated on the 2nd day in Hypoxia $(r=-0.66, p<.05)$ (Fig. 2A), 2nd night in Hypoxia $(r=-0.80, \mathrm{p}<.05)$ (Fig. 2B), and on the 2nd day in Exercise under Hypoxia $(r=-0.72, \mathrm{p}<.05)$ (Fig. 2C), 2nd night in Exercise under Hypoxia $(r=-0.89, p<.05)$ (Fig. 2D). For the Normoxia, the correlations were $(r=0.22)$ and for the Exercise under Normoxia $(\mathrm{r}-0.16)$ during the 2 nd night and 2 nd day respectively. Those correlations no were statistically significant $(p>.05)$.

The associations between PMC and $\mathrm{SaO} 2 \%$ in the total cohort were evaluated, as well as over both the total hypoxia exposure and total no hypoxia groups. The sum of the Hypoxia and Exercise under Hypoxia were negatively correlated during 2nd night $(r=-0.90, p<.05)$ (Fig. 2E), and during 2nd day $(r=-0.70, p<.05)$ (Fig. 2F). In the sum of the Normoxia and Exercise under Normoxia, there were not significant correlations during 2nd night or 2nd day respectively $(r=-0.22$ and $r=0.09, \mathrm{p}>.05)$.

Fig. 3 shows that associations between the PMC, SL, and SE were also performed. The PMC and SL were negatively correlated during 2nd night in Normoxia $(r=-0.79, p<.05)$ (Fig. 3A) and Exercise under Normoxia $(r=-0.81, \mathrm{p}<.05)$ (Fig. 3C), PMC and SL were positively correlated during 2nd night in Hypoxia $(r=0.76, \mathrm{p}<.05)$ (Fig. 3B) and Exercise under Hypoxia $(r=0.93, \mathrm{p}<.05)$ (Fig. 3D).

Fig. 4 shows that PMC and SE were positively correlated during 2nd night in Normoxia $(r=0.73, \mathrm{p}<.05)$ (Fig. 4A) and Exercise under Normoxia $(r=0.79, \mathrm{p}<.05)$ (Fig. 4C). The PMC and SE were negatively correlated during 2nd night in Hypoxia $(r=-0.75, \mathrm{p}<.05)$ (Fig. 4B) and Exercise under Hypoxia ( $r=-0.88, \mathrm{p}<.05$ ) (Fig. 4D).

Fig. 5 shows that in Normoxia the PMC was lower during 1st day in 
A

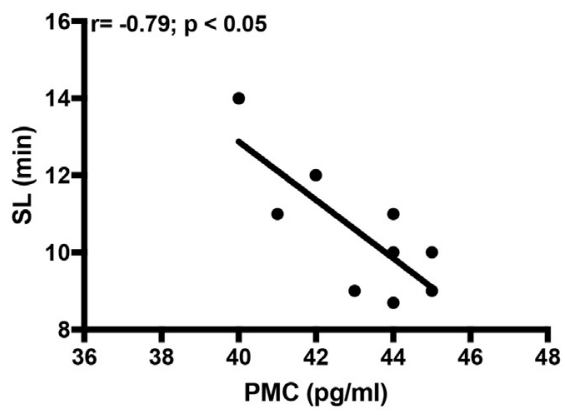

C

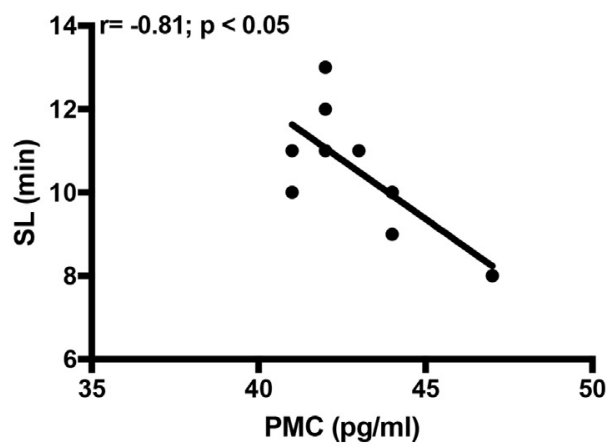

B

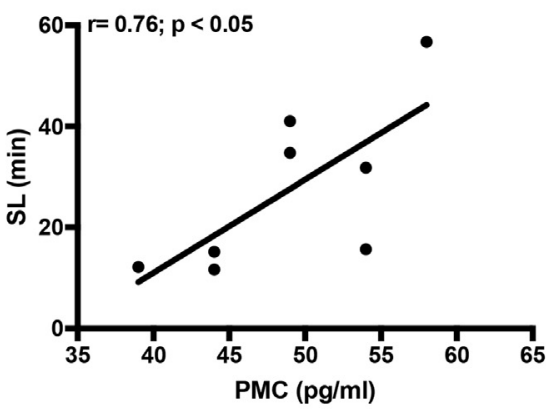

D

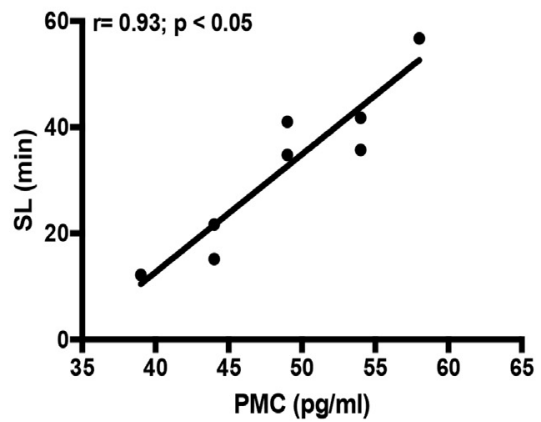

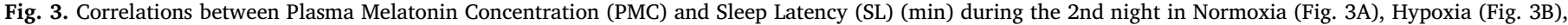
Exercise under Normoxia (Fig. 3C) and Exercise under hypoxia (Fig. 3D). Pearson's correlation coefficient (r) and p < 0.05.

relation to 1 st night $(p=.002)$ and during 2 nd day in relation to 2 nd night $(p=.001)$. In Hypoxia the PMC was lower during 1st day in relation to 1st night $(p=.004)$, during 2 nd night was high in relation to 1 st day $(p=.003)$ and during 2 nd day was higher in relation to 1 st day $(\mathrm{p}=.003)$. In Exercise under Normoxia, the PMC was lower during 1st day in relation to 1 st night $(p=.002)$ and during 2 nd day in relation to 2nd night $(p=.001)$. In Exercise under Hypoxia PMC was lower during 1 st day in relation to 1 st night ( $\mathrm{p}=.001$ ), on the other hand during 2 nd night was higher in relation to 1 st day $(p=.001)$. During 2 nd day was higher in relation to 1 st day $(\mathrm{p}=.004)$. The PMC was higher during

\section{A}

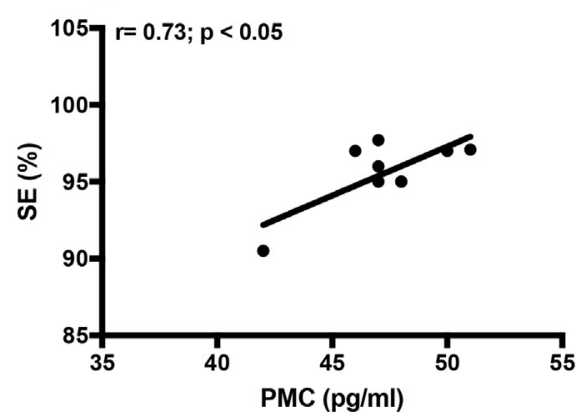

C

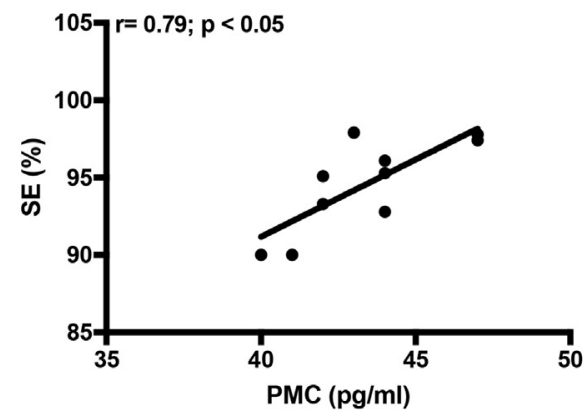

B

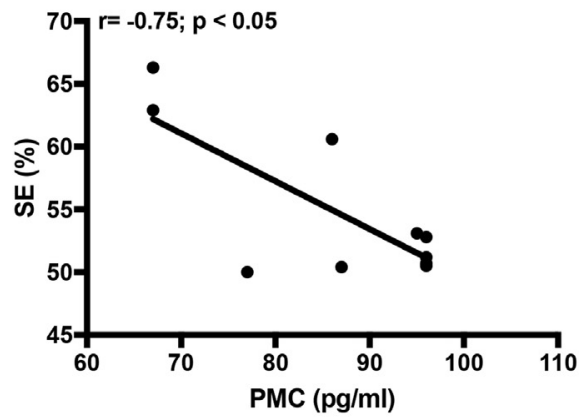

D

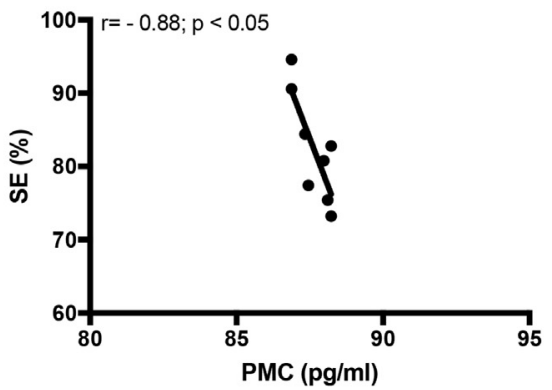

Fig. 4. Correlations between Plasma Melatonin Concentration (PMC) and sleep efficiency (SE) (\%) during the 2nd night in Normoxia (Fig. 4A), Hypoxia (Fig. 4B), Exercise under Normoxia (Fig. 4C) and Exercise under hypoxia (Fig. 4D). Pearson's correlation coefficient (r) and p $<0.05$. 


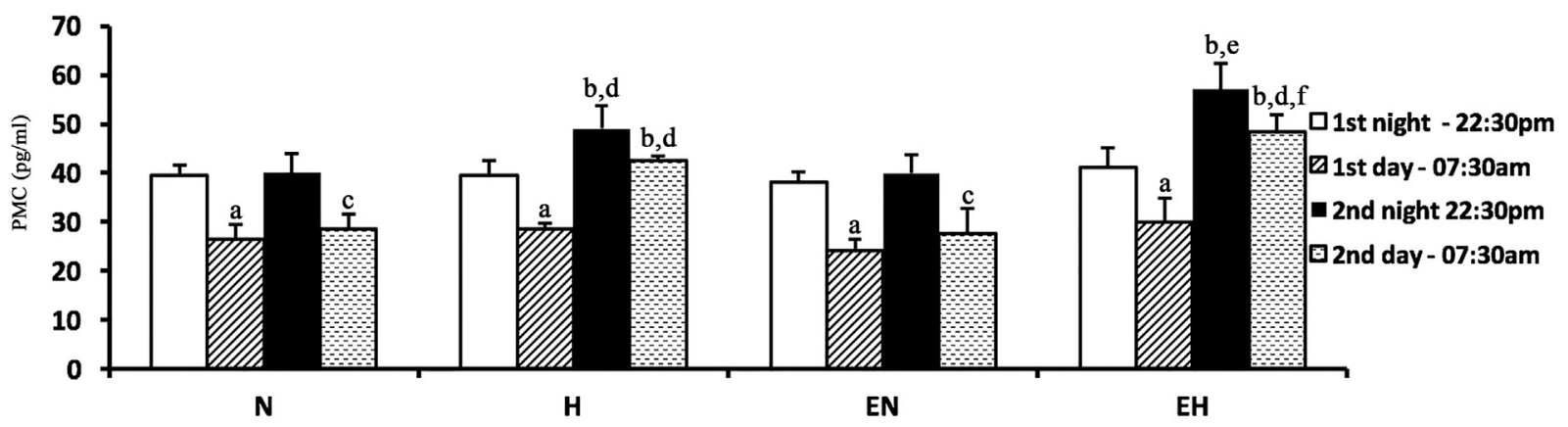

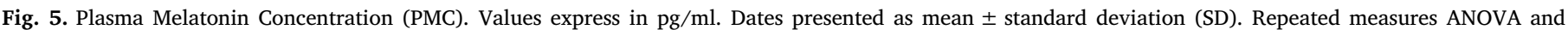

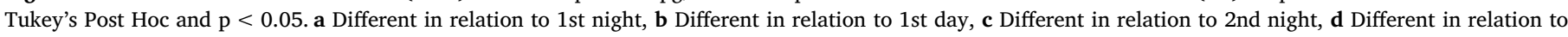

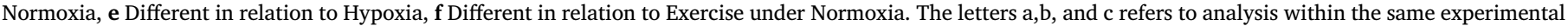
group, and d,f refers to analysis within the same time period.

2nd night in Hypoxia compared to Normoxia ( $\mathrm{p}=.004$ ), while during 2nd day it was higher in Hypoxia compared to 2nd day in Normoxia $(\mathrm{p}=.003)$. Finally, in Exercise under Hypoxia, the PMC was higher during 2nd night compared to Hypoxia $(p=.001)$, and during 2nd day compared to Normoxia $(p=.002)$ and in Exercise under Normoxia $(\mathrm{p}=.001)$.

Fig. 6 demonstrates the PMC in the delta between the 2nd and 1st nights. An increase was observed in Hypoxia compared to Normoxia $(\mathrm{p}=.001)$. In addition, it was observed an increase in Exercise under Hypoxia in relation to Hypoxia and Exercise under Normoxia $(p=.002)$.

Fig. 7A shows that during 2nd night, the SL (min) in Hypoxia was higher compared to Normoxia $(\mathrm{p}=.002)$. During 2nd night in Exercise under Normoxia the SL was reduced compared to Normoxia $(p=.003)$ and decreased in Exercise under Hypoxia compared to Hypoxia ( $p=.001)$. There was an increase in SL during 2 nd night compared to 1st night in Hypoxia $(p<.001)$, same occurred for Exercise under Hypoxia $(p=.002)$, already in Exercise under Normoxia there was a reduction during 2 nd night compared to 1st night. TST (min) in Hypoxia was reduced during 2nd night compared to Normoxia $(\mathrm{p}=.002)$ and compared to 1 st night in Hypoxia $(p=.009)$. It was increase in Exercise under Normoxia during 2nd night $(\mathrm{p}=.001)$ compared to Normoxia. In addition, the TST was lower during 2nd night in Exercise under Hypoxia compared to Hypoxia $(\mathrm{p}=.001)$ and Exercise under Normoxia ( $p=.006$ ) (Fig. 7B). SE (\%) in Hypoxia was decreased during 2nd night $(\mathrm{p}=.002)$ and 1 st night in Hypoxia $(p=.003)$ compared to Normoxia. In Exercise under Normoxia the 2nd night was higher than Hypoxia. Finally, during 2nd night in Exercise under Hypoxia were lower in comparison to Exercise under Normoxia $(p=.003)$ and higher than Hypoxia ( $\mathrm{p}=.003$ ) (Fig. 7C).

\section{Discussion}

In the present study, we observed that worsening sleep in hypoxia may be mediated by melatonin modification. Conversely, one hour of moderate physical exercise improved sleep onset latency, total sleep time, and sleep efficiency after $24 \mathrm{~h}$ of hypoxia. The hypoxia was similar to conditions experienced at an altitude of $4500 \mathrm{~m}$.

Melatonin, a synchronizer of circadian rhythms, represents one of the pivotal factors interrelating environmental influences (light, oxygen) with cellular responses. Chronic circadian misalignment can lead to pre-diabetic manifestations [39]. There is also a clear linkage between sleep disturbance, glucose deregulation, and predisposition to metabolic disorders [23].

Melatonin can act as a neuroprotective antioxidant in both normoxia and hypobaric hypoxia, which can prevent and counteract the deleterious effects of oxidative stress including neuronal cell death, reactive astrogliosis, and cognitive impairment [40]. Melatonin can stabilize and strengthen the coupling of circadian rhythms, particularly of core temperature and sleep-wake rhythms [41]. One hypothesis for the increase in melatonin in hypoxia observed in this study is that hypoxia causes a drop or oscillations in body temperature, which alter circadian rhythms and can lead to alterations in sleep, as observed in the present study in the $\mathrm{H}$ group [42,43]. This change in circadian rhythm may influence the melatonin plasmatic concentration, which can consequently deregulate sleep [44]. Conversely, in the EH group, there were improvements in sleep onset latency, total sleep time, and sleep efficiency compared to the $\mathrm{H}$ group. This may have occurred as hypoxia can increase the fragmentation of sleep, which may lead to greater secretion of melatonin to try to consolidate sleep and reduce fragmentation. However, the role of melatonin in adaptation in cases of

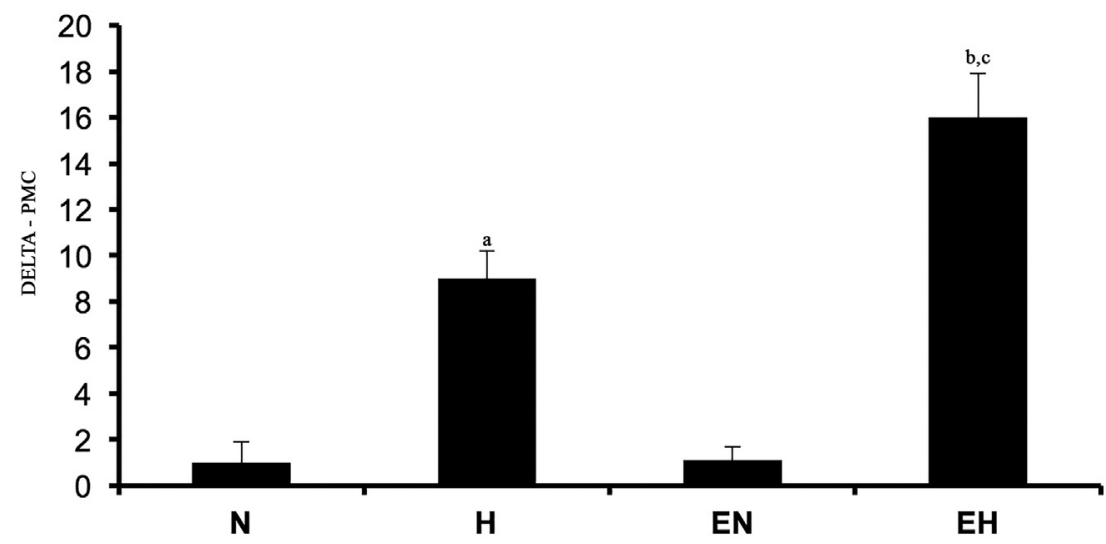

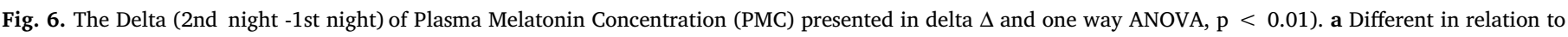
Normoxia, b Different in relation to Hypoxia, c Different in relation to Exercise under Normoxia. 

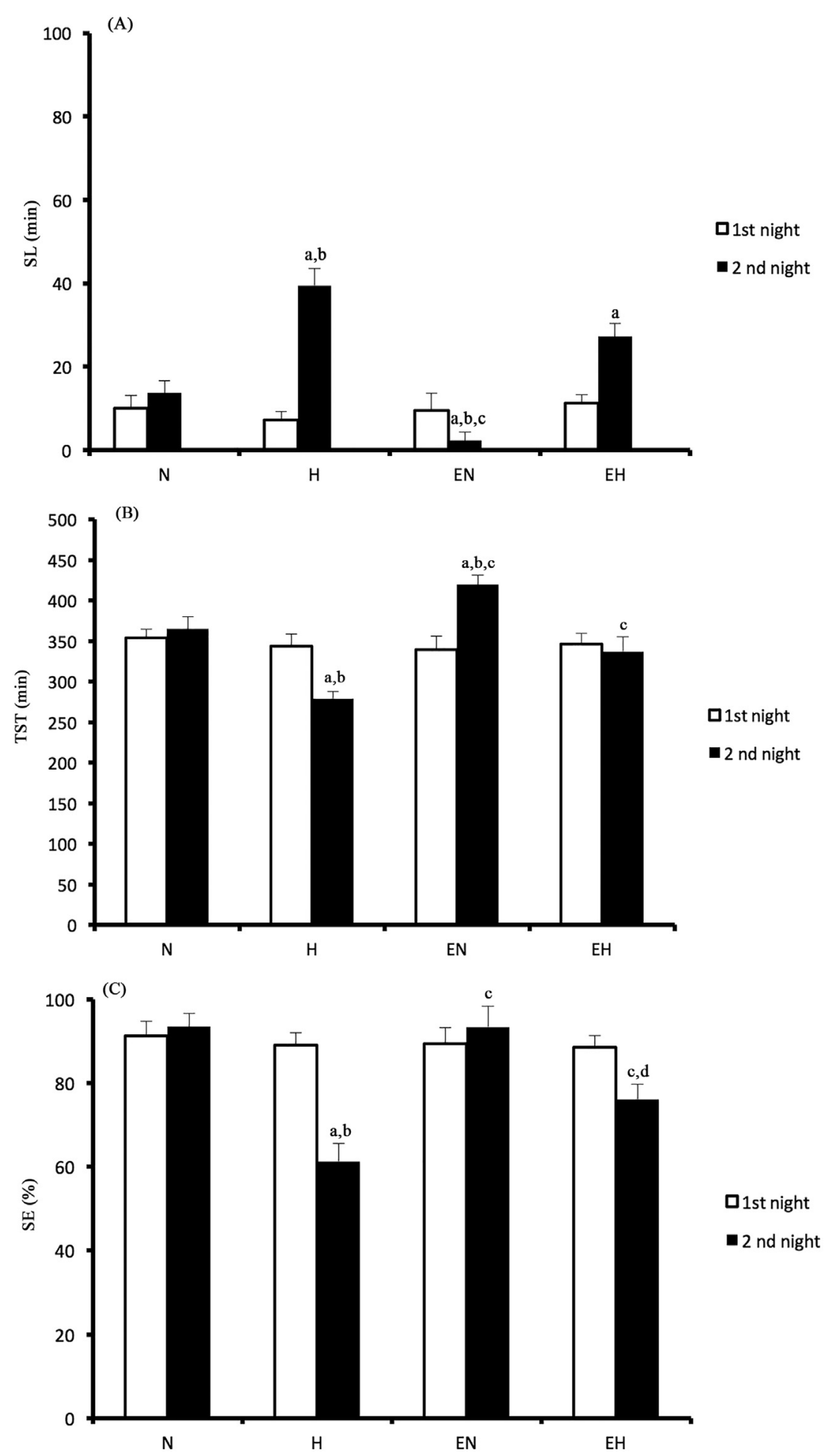

Fig. 7. Shows Sleep Latency (SL) (min) (Figure 7A), Total Sleep Time (TST) (min) (Figure 7B) and sleep efficiency (SE) (\%) (Figure 7C). Repeated measures ANOVA and Tukey's Post Hoc, $\mathrm{p}<0.05$. a Different in relation to 1stnight, $\mathbf{b}$ Different in relation to normoxia, $\mathbf{c}$ Different in relation to hypoxia, $\mathbf{d}$ Different in relation to exercise under normoxia.

chronic exposure to hypoxia should be investigated.

One hypothesis is that physical exercise modulates circadian rhythm, thus improving the quality of sleep [45]. Increased levels of melatonin can decrease alertness at work and affect safety [46]. Driving at night, particularly at higher altitudes, may increase the risk of accidents if melatonin secretion is not suppressed by adequate lighting, including short-wavelength light between approximately 440 and $470 \mathrm{~nm}$, which is most effective at stimulating the human circadian rhythm system to reduce plasma levels of melatonin [47,48]. Nocturnal melatonin elevation tends to persist, under hypoxic conditions, in the early morning, as observed in the present study (Fig. 3). Eckel et al. [49] reported that a delay in nocturnal melatonin remission under hypoxic conditions may represent an additional factor promoting sleepiness and accidents.

Adaptive responses to aerobic exercise also seem to require melatonin [50]. It would be of interest to establish whether the acute effects 
of hypoxia and physical exercise on melatonin, as observed in the present study, persist in the long term at high altitude. Physical exercise may improve sleep as observed in this study; however, it was likely not mediated by melatonin, which remained high. The correct conclusion may be as follows: there may have been a loss of sensitivity to hypothalamic melatonin or other mechanisms still unknown that were more intense than melatonin. Therefore, hypoxia may decrease melatonin sensitivity and physical exercise cannot reverse this decrease. The mechanism of action of physical exercise in this study remains unknown.

In the present study, melatonin was measured in subjects exposed to normoxic hypoxia under controlled laboratory conditions to discern between the effects of hypoxia and exercise. In apparent contrast to the present results obtained on exposure to normobaric hypoxia (Fig. 3), a condition of hypobaric hypoxia has been found to decrease nocturnal melatonin [26]. This conflict can be resolved on the basis of differences in the overall pressure being applied. As opposed to normobaric hypoxia, the hypobaric condition affects not only the supply of oxygen but also that of other gases, such as nitric oxide [51]. Interestingly, a decrease in nitric oxide affects circadian rhythmicity [52]. Whether melatonin is involved in the nitric oxide induced alteration of circadian rhythmicity remains to be elucidated.

The increase in melatonin observed in the present study could be attributed to hypoxia as the hormone levels were negatively correlated with $\mathrm{SaO}_{2} \%$ only in the $\mathrm{H}$ and $\mathrm{EH}$ groups (Fig. 2). Hypoxia is defined as the lack of oxygen in relation to the demand by ATP [53]. In our study, the aerobic physical exercise performed by the EH group appeared to potentiate the effect on melatonin observed in the $\mathrm{H}$ group (Fig. 3). Studies show that in the hypoxia condition there is an increase in oxidative stress and, consequently, in the production of ROS [54,55]. In addition, higher intensity physical exercise may increase oxidative stress and contribute to ROS formation resulting from metabolic activity or damage to skeletal muscle [56]. Oxidative stress may contribute to the increase in ROS in the condition of hypoxia without physical effort, however, when added to exercise can potentialize the increase [57]. Any condition involving hypoxia and reoxygenation involves the possibility of tissue damage by reactive oxygen species (ROS). Metabolism influences clock function via redox status [58]. Thus, the increase in nocturnal melatonin in the $\mathrm{H}$ and $\mathrm{E}$ groups (Fig. 3) may also be observed in the context of the well-known antioxidant effect of melatonin [12,59]. The increase in melatonin in hypoxia may therefore be interpreted as a protective effect induced by the lack of oxygen, as a possible countermeasure suppressing concomitant oxidative stress. Another documented protective effect of melatonin refers to neurohumoral stress. Peripheral effects of exacerbated sympathetic activity are indeed mitigated by melatonin [13].

\section{Conclusion}

In summary, we found that, in relation to sleep, one hour of moderate physical exercise improved sleep onset latency, total sleep time, and sleep efficiency, after $24 \mathrm{~h}$ of hypoxia. Our main conclusion is that normobaric hypoxia, equivalent to oxygen availability at an altitude of $4500 \mathrm{~m}$, acutely increases melatonin. Diurnal remission of the nocturnal increase in melatonin seems to be delayed by hypoxia and to an even greater extent if acting together with exercise. However, studies with other experimental designs should be performed, taking into account the circadian variation of melatonin concentration. Thus, it would be interesting to perform serial evaluations including 6 to 8 measurements throughout the day. In addition, it would also be possible to evaluate whether the effects of the exercise remain at other times of the day.

\section{Acknowledgments}

All authors thank the Associacão Fundo de Incentivo a Psicofarmacologia - AFIP), Centros de Pesquisa, Expansão e Difusão do Instituto do Sono-(CEPID/SONO), Fundacão de Amparo a Pesquisa do Estado de São Paulo-(FAPESP) $n^{\circ}(2012 / 09236-4)$ and (2010/ 19026-1), Universidade Federal de São Paulo-(UNIFESP) e Centro de Estudos em Psicobiologia e Exercício-(CEPE).

\section{References}

[1] Gandhi AV, Mosser EA, Oikonomou G, Prober DA. (2015). Melatonin is required for the circadian regulation of sleep. Neuron, 18;85(6):1193-9.

[2] F. Luchetti, B. Canonico, M. Betti, M. Arcangeletti, F. Pilolli, M. Piroddi, L. Canesi, S. Papa, F. Galli, Melatonin signaling and cell protection function, FASEB J. 24 (2010) 3603-3624.

[3] J. Richards, M.L. Gumz, Advances in understanding the peripheral circadian clocks, FASEB 26 (2012) 3602-3613.

[4] J. Emens, A.J. Lewy, K. Yuhas, A.R. Jackman, K.P. Johnson, Melatonin entrains free-running blind individuals with circadian periods led than 24 hours, Sleep 29 (2006) A62.

[5] B. Jung-Hynes, W. Huang, R.J. Reiter, N. Ahmad, Melatonin resynchronizes dysregulated circadian circuitry in human prostate cancer cells, J. Pineal Res. 49 (2010) 60-68.

[6] P.F. Innominato, A.S. Lim, O. Palesh, M. Clemons, M. Trudeau, A. Eisen, C. Wang, A. Kiss, K.I. Pritchard, G.A. Bjarnason, The effect of melatonin on sleep and quality of life in patients with advanced breast cancer, Support Care Cancer 24 (3) (2015) 1097-1105.

[7] C. Veneroso, M.J. Tunon, J. González-Gallego, O.S. Collado, Melatonin reduces cardiac inflammatory injury induced by acute exercise, J. Pineal Res. 47 (2009) $184-191$.

[8] M. Tare, H.C. Parkington, E.M. Wallace, A.E. Sutherland, R. Lim, T. Yawno, H.Á. Coleman, G. Jenkin, S. Miller, Maternal melatonin administration mitigates coronary stiffness and endothelial dysfunction, and improves heart resilience to insult in growth restricted lambs, J. Physiol. 592 (12) (2014) 2695-2709.

[9] R.J. Reiter, D.X. Tan, M.D. Maldonado, Melatonin as an antioxidant: physiology versus pharmacology, J. Pineal Res. 39 (2005) 215-216.

[10] R.J. Reiter, D.-X. Tan, L.C. Manchester, S.D. Paredes, J.C. Mayo, R.M. Sainz Melatonin and reproduction revisited, Biol. Reprod. 81 (2009) 445-456.

[11] K.G. Akbulut, B. Gonul, H. Akbulut, Exogenous melatonin decreases age-induced lipid peroxidation in the brain, Brain Res. 1238 (2008) 31-35.

[12] D.X. Tan, R. Hardeland, L.C. Manchester, S.D. Paredes, A. Korkmaz, R.M. Sainz, J.C. Mayo, L. Fuentes-Broto, R.J. Reiter, The changingbiological roles ofmelatoninduringevolution: fromanantioxidanttosignalsofdarkness, sexual selectionand fitness, Biol. Rev. Camb. Philos. Soc. 85 (3) (2010 Aug) 607-623.

[13] M.D. Muller, C.L. Sauder, C.A. Ray, Melatonin attenuates the skin sympathetic nerve response to mental stress, Am. J. Physiol. Heart. Circ. Physiol. 305 (2013) H1382-H1386.

[14] B. Jung-Hynes, W. Huang, R.J. Reiter, N. Ahmad, Melatoninresynchronizesdysregulatedcircadianrhythmcircuitry in humanprostatecancercells, J. Pineal Res. 49 (1) (2010) 60-68.

[15] A. Bukowska, Anticarcinogenic role of melatonin - potential mechanisms, Med. Pr. 62 (2011) 425-434.

[16] A. Cutando, A. López-Valverde, S. Arias-Santiago, J. De Vicente, R. Gómez de Diego, Anticancer Res. 32 (2012) 2747-2754.

[17] R. Santoro, F. Mori, M. Marani, G. Grasso, A.M. Cambria, G. Blandino, P. Muti, S. Strano, Blockage of melatonin receptors impairs p53-mediated prevention of DNA damage accumulation, Carcinogenesis 34 (2013) 1051-1061.

[18] S.M. Hill, V. Belancio, R.T. Dauchy, S. Xiang, S. Brimer, L. Mao, A. Hauch, P.W. Lundberg, W. Summers, L. Yuan, T. Frasch, D.E. Blask, Melatonin: an inhibitor of breast cancer, Endocr. Relat. Cancer 22 (2015) R183-R204.

[19] C. Saiki, J.P. Mortola, Hypoxia abolishes the morning-night differences of metabolism and ventilation in 6-day-old rats, Can. J. Physiol. Pharmacol. 73 (1995) 159-164.

[20] J.P. Mortola, E.L. Seifert, Hypoxic depression of circadian rhythms in adult rats, J. Appl. Physiol. 88 (2000) 365-368.

[21] M. Vargas, D. Jimenez, F. León-Velarde, J. Osorio, J.P. Mortola, Circadian patterns in men acclimatized to intermittent hypoxia, Respir. Physiol. 126 (2001) 233-243.

[22] G. Bosco, A. Ionadi, S. Panico, F. Faralli, R. Gagliardi, P. Data, J.P. Mortola, Effects of hypoxia on the circadian patterns in man, High Alt. Med. Biol. 4 (2003) 305-318.

[23] S.M. Bailey, U.S. Udoh, M.E. Young, Circadian regulation of metabolism, J. Endocrinol. 222 (2014) R75-R96.

[24] F. Kong, S. Liu, Q. Li, L. Wang, Sleep architecture in partially acclimatized lowlanders and native Tibetan Sat 3800 meter altitude: what are the differences? High Alt. Med. Biol. 16 (3) (2015 Sep) 223-229.

[25] C. Kaur, K.N. Srinivasan, J. Singh, C.M. Peng, E.A. Ling, Plasma melatonin, pinealocytemorphology, andsurfacereceptors/antigenexpressiononmac- rophages/microglia in the pineal glandfollowing a high-altitude exposure, J. Neurosci. Res. 67 (2002) 533-543.

[26] O. Coste, M. Beaumont, D. Bate'jat, P. Van Beers, H. Charbuy, Y. Touitou, Hypoxicdepressionofmelatoninsecretionaftersimulatedlongdurationflight in man, $\mathrm{J}$. Pineal Res. 37 (2004) 1-10.

[27] K. Krauchi, C. Cajochen, A. Wirz-Justice, Circadian and homeostatic regulation of core body temperature and alertness in humans: what is the role of melatonin, in: K. Honma, S. Honma (Eds.), Circadian Clocks and Entrainment, 1998 Hokkaido Univ. Press, Sapporo, 1998, pp. 131-145. 
[28] H. Lee, S. Kim, D. Kim, Effects of exercise with or without light exposure on sleep quality and hormone reponses, J. Exerc. Nutrition Biochem. 18 (3) (2014) 293-299.

[29] G. Escames, G. Ozturk, B. Baño-Otálora, M.J. Pozo, J.A. Madrid, R.J. Reiter, E. Serrano, M. Concepción, D. Acuña-Castroviejo, Exercise and melatonin in humans: reciprocal benefits, J. Pineal Res. 52 (1) (2012) 1-11.

[30] D.B. Carr, S.M. Reppert, B. Bullen, et al., Plasma melatonin increases during exercise physical in women, J. Clin. Endocrinol. Metab. 53 (1981) 224-225.

[31] G.S. Skrinar, B.A. Bullen, S.M. Reppert, et al., Melatonin response toexercise training in women, J. Pineal Res. 7 (2) (1989) 185-194.

[32] P. Monteleone, M. Maj, M. Fusco, C. Orazzo, D. Kemail, Physical exercise at night blunts the nocturnal increase of plasma melatonin level in healthy human, Life Sci. 1990 (1989-1995) 47.

[33] P. Monteleone, M. Maj, A. Fuschino, et al., Physical stress in the middle of the dark phase does not affect light-depressed plasma melatonin levels in humans, Neuroendocrinology 55 (1992) 367-371.

[34] A.N. Elias, A.F. Wilson, M.R. Pandian, et al., Melatonin and gonadotropin secretion after acute exercise in physically active males, Eur. J. Appl. Physiol. Occup. Physiol. 66 (1993) 357-361.

[35] T. Miyazaki, S. Hashimoto, S. Masubuchi, et al., Phase-advance shifts of human circadian pacemaker are accelerated by daytime physical exercise, Am. J. Physiol. Regul. Integr. Comp. Physiol. 281 (2001) R197-R205.

[36] H. Frisch, F. Waldhauser, T. Waldhör, A. Müllner-Eidenböck, P. Neupane, K. Schweitzer, (2004) increase in 6-hydroxymelatonin excretion in humans during ascent to high altitudes, J. Clin. Endocrinol. Metab. 89 (9) (2004) 4388-4390.

[37] J.A. Harris, F.G. Benedict, A Biometric Study of Basal Metabolism in Man, Publication No. 279 of the Carnegie Institution of Washington, Washington, DC, (1919).

[38] C. Iber, S. Ancoli-Israel, A. Chesson, S.F. Quan, For the American Academy of Sleep Medicine. The AASM Manual for the Scoring of Sleep and Associated Events: Rules, Terminology and Technical Specifications, American Academy of Sleep Medicine, Westchester, Ill, 2007.

[39] O.M. Buxton, S.W. Cain, S.P. O'Connor, J.H. Porter, J.F. Duffy, W. Wang, C.A. Czeisler, S.A. Shea, Adverse metabolic con sequences in humans of prolonged sleep restriction combined with circadian disruption, Sci. Transl. Med. 4 (129) (2012) 129ra43.

[40] C. Vornicescu, B. Boșca, D. Crişan, S. Yacoob, N. Stan, A. Filip, A. Şovrea, Neuroprotective effect of melatonin in experimentally induced hypobaric hypoxia, Rom. J. Morphol. Embryol. 54 (4) (2013) 1097-1106.

[41] B. Claustrat, J. Leston, Melatonin: physiological effects in humans, Neurochirurgie $61(2-3)(2015) 77-84$.

[42] O. Coste, P. Van Beers, Y. Touitou, Hypoxia-inducedchanges in recoverysleep, core bodytemperature, urinary 6-sulphatoxymelatonin andfree cortisol after a simulatedlong-durationflight, J. Sleep Res. 18 (4) (2009) 454-465.

[43] J.P. Mortola, Gender and the circadian pattern of body temperature in normoxia and hypoxia, Respir. Physiol. Neurobiol. (2016) (pii: S1569 9048(16)30221-X).

[44] H.H. Kozak, M. Boysan, A.U. Uca, A. Aydın, İ. Kılınç, E. Genç, M. Altaş, D.C. Güngör, K. Turgut, N. Özer, Sleep quality, morningness- eveningnesspreference,mood profile, and levels of serum melatonin in migraine patients: a case-control study, Acta Neurol. Belg. 117 (1) (2017) 111-119.

[45] D.S. Hwang, H.B. Kwak, I.G. Ko, S.E. Kim, J.J. Jin, E.S. Ji, H.H. Choi, O.Y. Kwon, Treadmill exercise improves memory function depending on circadian rhythm changes in mice, Int. Neurourol. J. 20 (Suppl. 2) (2016) S141-S149.

[46] M.G. Figueiro, L. Sahin, B. Wood, B. Plitnick, Light at night and measures of alertness and performance: implications for shift workers, Biol. Res. Nurs. 18 (1) (2016) 90-110.

[47] G. Brainard, J. Hanifin, J. Greeson, B. Byrne, G. Glickman, E. Gerner, et al., Action spectrum for melatonin regulation in humans: evidence for a novel circadian photoreceptor, J. Neurosci. 21 (2001) 6405-6412.

[48] K. Thapan, J. Arendt, D.J. Skene, An action spectrum for melatonin suppression: evidence for a novel non-rod, non-cone photoreceptorsystem in humans, J. Physiol 535 (2001) 261-267.

[49] R.H. Eckel, C.M. Depner, L. Perreault, R.R. Markwald, M.R. Smith, A.W. McHill, J. Higgins, E.L. Melanson, K.P. Wright Jr., Morning circadian misalignment during short sleep duration impacts insulin sensitivity, Curr. Biol. 25 (22) (2015) 3004-3010.

[50] Mendes, et al., Adapations of the aging animal to exercise: role of daily supplementation with melatonin, J. Pineal Res. 55 (3) (2013) 229-239.

[51] R. Faiss, V. Pialoux, C. Sartori, C. Faes, O. Dériaz, G.P. Millet, Ventilation, oxidative stress, and nitric oxide in hypobaric versus normobaric hypoxia, Med. Sci. Sports Exerc. 45 (2013) 253-260.

[52] Kunieda T, Minamino T, Miura K, Katsuno T, Tatena T, Miyauchi H, Kaneko S, Bradfield CA, Fitzgerald GA, KomuroI (2008). Reduced nitric oxide causes age-associated impairment of circadian rhytmicity. Circ. Res., 102:607-614.

[53] R.J. Connett, C.R. Honig, T.E. Gayeski, G.A. Brooks, Defining hypoxia: a system view of VO2, glycolysis, energetics and intracellular PO2, J. Appl. Physiol. 68 (1990) 833-842.

[54] P. Himadri, S.S. Kumari, M. Chitharanjan, S. Dhananjay, Role of oxidative stress and inflammation in hypoxia-induced cerebral edema: a molecular approach, High Alt. Med. Biol. 11 (3) (2010) 231-244 Fall.

[55] H. Hoppeler, M. Vogt, E.R. Weibel, M. Flück, Response of skeletal muscle mitochondria to hypoxia, Exp. Physiol. 88 (1) (2003) 109-119.

[56] S. Borges Lda, A. Dermargos, E.P. da Silva Junior, E. Weimann, R.H. Lambertucci, E. Hatanaka, Melatonin decreases muscular oxidative stress andinflammationinducedbystrenuousexerciseandstimulatesgrowthfactorsynthesis, J. Pineal Res. 58 (2) (2015) 166-172.

[57] Askew EW. Work at high altitude and oxidative stress: antioxidant nutrients. Toxicology, 2002, 15;180(2):107-19.

[58] K. Yoshii, S. Ishijima, I. Sagami, Effects of NAD(P)H and its derivatives on the DNA binding activity of NPAS2, a mammalian circadian transcription factor, Biochem. Biophys. Res. Commun. 437 (3) (2013) 386-391.

[59] A. Wakatsuki, Y. Okatani, N. Ikenoue, K. Shinohara, K. Watanabe, T. Fukaya, Melatoninprotectsagainstoxidizedlow-densitylipoprotein-inducedinhibitionofnitric oxide production in human umbilical artery, J. Pineal Res. 31 (3) (2001) 281-288. 